\title{
Efecto del entrenamiento de la musculatura inspiratoria con válvula umbral sobre la capacidad funcional en mujeres físicamente activas mayores de 60 años
}

\author{
Effect of inspiratory muscle training with threshold valve on functional capacity of physically active women who are \\ older than 60 years of age
}

\author{
Alexis Espinoza-Salinas $1.2,4 *$ orcid.org/0000-0001-9709-2850 \\ Giovanny Arenas-Sánchez ${ }^{1,4}$ orcid.org/0000-0002-3764-9378 \\ Roberto Fernández-García' orcid.org/0000-0003-0245-7461 \\ Omar Ravanales-Astudillo' orcid.org/0000-0002-4458-4541 \\ José González-Jurado 3 orcid.org/0000-0003-2222-6089
}

Edson Zafra-Santos' orcid.org/0000-0001-9583-1373

\begin{abstract}
Laboratorio de Fisiología del Ejercicio, Escuela de Kinesiología, Universidad Santo Tomás. Santiago, Chile
Doctorado en Ciencias de la Actividad Física y Deporte, Universidad Pablo de Olavide. Sevilla, España

Facultad de Ciencias del Deporte, Universidad Pablo de Olavide. Sevilla, España

Centro de Ejercicio Adaptado, YMCA. Santiago, Chile
\end{abstract}

Introducción: Los cambios fisiológicos asociados al envejecimiento generan una serie de modificaciones funcionales, destacando una disminución en la capacidad respiratoria. En este sentido, una estrategia alternativa para mejorar tal condición podría ser el entrenamiento de la musculatura inspiratoria (EMI). Objetivo: Analizar el efecto del EMI con válvula umbral, sobre la capacidad funcional en mujeres físicamente activas mayores de 60 años. Materiales y métodos: Se realizó un EMI con válvula umbral durante 4 semanas, sobre un grupo experimental (GE; n:10), contrastado con un grupo control (GC; n:5). Se valoraron las siguientes variables hemodinámicas y antropométricas: peso, talla, índice de masa corporal (IMC), lactato, doble producto y capacidad funcional, analizando los cambios pre y post entrenamiento. Resultados: en el grupo GE disminuye post intervención la concentración de lactato y la escala de percepción del esfuerzo; 3,16 $\pm 0,51$ a 2,5 $\pm 0,39$ y 5,56 $\pm 1,81$ a 4 42 , respectivamente. En el mismo grupo se incrementan los valores de

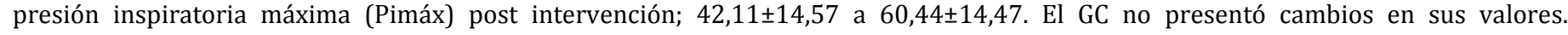
Conclusión: No se evidencian cambios favorables en los metros recorridos post intervención, sin embargo, el EMI mostró una disminución sobre la concentración de lactato post ejercicio, lo que podría identificar un retraso en la aparición de la fatiga.

Palabras clave: Presión inspiratoria máxima; entrenamiento del músculo respiratorio; lactato; tests función respiratoria; adulto mayor. (Fuente: DeCS, Bireme).

\section{Abstract}

Introduction: Physiological changes associated with aging generate a series of functional modifications, mainly a decrease in respiratory capacity. In this regard, an alternative strategy to improve such a condition could be inspiratory muscle training (IMT). Objective: To analyze the effect of IMT with threshold valve on the functional capacity of physically active women who are older than 60 years of age. Materials and methods: IMT with threshold valve was carried out over a period of 4 weeks, comparing data from the experimental group (GE; n:10) to the control group (GC; n:5). The following hemodynamic and anthropometric variables were assessed: weight, height, body mass index (BMI), lactate, double product (DP) and functional capacity. Pre and post training changes were evaluated. Results: The GE group showed a decrease in both post-intervention lactate concentration (from $3.16 \pm 0.51$ to $2.5 \pm 0.39$ ) and effort perception scale (from $5.56 \pm 1.81$ to $4 \pm 2$ ). On the other hand, this group experienced a post-intervention increase in maximal inspiratory pressure (MIP) from $42.11 \pm 14.57$ to $60.44 \pm 14.47$. The GC group did not present changes in its values. Conclusion: Although no evidence was found regarding favorable changes in the walked distance post-intervention, IMT induced a reduction in post-exercise lactate concentration, which could indicate a delay of onset of fatigue.

Key words: Maximal inspiratory pressure; respiratory muscle training; lactate; respiratory function tests; elderly. (Source: DeCS, Bireme). 


\section{Introducción}

El envejecimiento de la población es un hecho a nivel global, actualmente alrededor del $7 \%$ de la población mundial es mayor de 65 años, y en los países desarrollados este porcentaje es aún mayor $(15 \%)^{(1)}$. En Chile en el año 2012, existía una cantidad de adultos mayores (AM) superior al $15 \%$ de la población total, y se espera que para el 2025 la población mayor de 60 años constituya un $20 \%$, lo que superará el porcentaje de población menor de 15 años(2). En este contexto, los cambios fisiológicos asociados al envejecimiento generan una serie de modificaciones funcionales, destacando una disminución en la capacidad funcional (CF), que se vincula al sedentarismo. De acuerdo con esto, sólo un $7 \%$ de los AM realiza actividad física de mediana intensidad, lo que se traduce en una disminución progresiva de la $\mathrm{CF}^{(3)}$.

La práctica regular de actividad física se asocia a beneficios en el control de enfermedades cardiometabólicas y musculoesqueléticas(4). El tipo de ejercicio físico realizado en esta población se orienta en la participación social activa, incorporando ejercicios aeróbicos de baja o mediana intensidad y trabajos de fortalecimiento muscular(5). En este sentido, surge un método alternativo de entrenamiento que se ha aplicado tanto a deportistas como a personas con enfermedades cardiorrespiratorias. Este método es conocido como inspiratory muscle training, o entrenamiento de la musculatura inspiratoria (EMI), que consiste en la utilización de un dispositivo inhalador que otorga resistencia al flujo de aire inspiratorio; siendo de fácil utilización y una alta aplicabilidad por parte del usuario(6).

El objetivo del estudio fue analizar el efecto del entrenamiento de la musculatura inspiratoria con la válvula umbral sobre la capacidad funcional en mujeres físicamente activas mayores de 60 años pertenecientes la Asociación Cristiana de Jóvenes (YMCA) Santiago de Chile.

\section{Materiales y métodos}

El estudio constó de 15 mujeres AM, sanas, sin antecedentes mórbidos diagnosticados ni reportados, que no presentaran alguna lesión o enfermedad que les impidiera caminar. 10 individuos se clasificaron como el grupo entrenamiento (GE; edad $=74,22 \pm$ 7,07 años), y el grupo control estaba conformado por 5 individuos (GC; edad = 74,8 \pm 7,98 años) (Tabla 1 ). El muestreo fue realizado por conveniencia, generando una distribución desigual asociada a la cantidad de participantes que cumplían los criterios de ingreso. Los individuos participaban en un programa de ejercicio y salud pertenecientes al Centro de Ejercicio Adaptado (CEA). El programa consistió en la realización de ejercicio aeróbico continuo, flexibilidad, coordinación, fuerza y estabilización lumbo-pélvica, supervisado por educadores físicos, kinesiólogos y médicos especialistas. Todos los participantes son físicamente activos ( $\geq 150$ minutos de actividad física de intensidad moderada o vigorosa por semana). Los criterios de exclusión fueron: presión arterial sobre 130/85mmHg no controlada y/o alguna patología músculoesquelética, metabólica o sistémica aguda o crónica que les impidieran realizar el protocolo de ejercicios.

Tabla 1. Características antropométricas de los participantes

\begin{tabular}{|c|c|c|}
\hline Variable & Grupo control & Grupo entrenamiento \\
\hline Edad, años & $74,8 \pm 7,98$ & $74,22 \pm 7,07$ \\
\hline Peso, Kg & $69,8 \pm 6,91$ & $70,44 \pm 9,99$ \\
\hline Altura, metros & $160,2 \pm 5,07$ & $157,4 \pm 7,04$ \\
\hline $\mathrm{IMC}, \mathrm{Kg} / \mathrm{mts}^{2}$ & $27,24 \pm 2,90$ & $28,3 \pm 2,52$ \\
\hline
\end{tabular}

\section{Procedimiento}

El estudio se realizó en el Laboratorio de Fisiología del Ejercicio de la Universidad Santo Tomás, sede Santiago, en conjunto con el YMCA. Los sujetos fueron distribuidos en dos grupos mediante aleatorización simple. El 100\% de los sujetos se mantuvieron en el programa de ejercicios del CEA por 4 semanas y 5 veces a la semana. El grupo GE realizó el entrenamiento de la musculatura inspiratoria en conjunto con el programa de ejercicios (Figura 1). El GC sólo realizó su programa habitual de ejercicios. Se controló la frecuencia cardiaca, presión arterial, y percepción de esfuerzo a través de la escala Borg con fines de seguridad y control, así como la correcta hidratación de los participantes y se evitó el consumo de alimentos en las dos horas previas a la medición. 


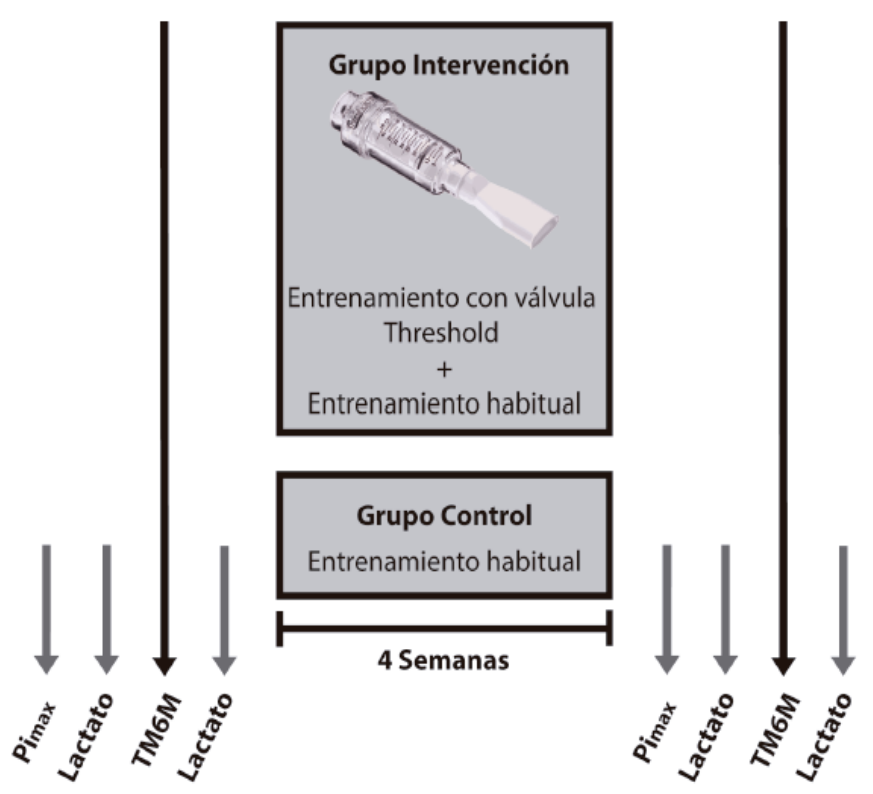

Figura 1. Diseño experimental usado en la investigación

\section{Presión Inspiratoria Máxima (Pimax)}

Durante la mañana (entre las 8 y 10 horas), se realizó la medición de la presión inspiratoria máxima (Pimax), dos días antes y después de las 4 semanas que duró la intervención. Previo a la medición de la Pimax, las participantes debían estar durante 10 minutos en reposo. Para su implementación se utilizó un equipo de pimometría (CareFusion modelo Micro RPM, USA), que registra la presiones inspiratoria máximas permitiendo evaluar la fuerza de los músculos respiratorios, y con estos datos graduar la válvula Threshold al porcentaje buscado. La prueba se realizó con el sujeto sentado, posicionándose una naricera para obstruir esa vía aérea, con el tórax y cuello en posición erguida y con ambos pies apoyados sobre el piso. Posteriormente, se le coloco una boquilla con filtro, sellada con los labios. Se le solicitó que realice una exhalación suave, pero completa y que luego inhalara fuerte y rápido durante aproximadamente 2 segundos. Se obtuvo 3 intentos reproducibles (menos de $10 \%$ de diferencia entre los dos de mayor valor), en caso de no alcanzar valores esperados se realizó hasta 8 intentos, con pausas de 1 minuto entre cada intento( ${ }^{(7)}$.

\section{Lactacidemia}

El lactato sanguíneo fue registrado pre y post evaluación del test de marcha de 6 minutos (TM6M), tanto al inicio como al final de la intervención de 4 semanas con la vávula Threshold. La condición previa (reposo) se consiguió con el sujeto en decúbito supino durante 10 minutos, posteriormente desde sedente se efectuó una punción a nivel del ortejo del dedo anular derecho para obtener una muestra de sangre capilar para analizar con equipo Accutrend Plus $^{\circledR}$ (Roche, Suecia), el procedimiento fue repetido inmediatamente finalizado el test de marcha de 6 minutos.

\section{Test de marcha 6 minutos (TM6M)}

La valoración de la capacidad funcional aeróbica se cuantificó con el TM6M, test submáximo que expresa la distancia recorrida durante 6 minutos en una superficie plana delimitada cada 30 metros. Se controló presión arterial, frecuencia cardiaca, saturación de oxígeno, escala de Borg y doble producto antes y después de la prueba. Previo al test, se verificó que los participantes no hubiesen realizado esfuerzo físico extenuante. Todos los sujetos debieron permanecer en reposo por lo menos 15 minutos antes de realizar el TM6M. Por último, se les indicó que no consumieran ningún tipo de estimulante de la frecuencia cardiaca, los días en que se realizó el test(8).

\section{Entrenamiento de la musculatura inspiratoria}

Para realizar el entrenamiento de la musculatura inspiratoria (EMI) se utilizó la válvula umbral. El GE realizó 30 esfuerzos respiratorios al 60\% del Pimax, en aproximadamente 10 a 15 minutos, una vez al día, 5 veces a la semana, durante 4 semanas, siendo a elección propia los 5 días de la semana que la persona quiso realizar las sesiones. La inspiración se realizó desde el volumen residual hasta la capacidad inspiratoria máxima, manteniendo el aire por 3 segundos y luego exhalando. Se reguló la intensidad una vez a la semana, de acuerdo a la progresión de la Pimax. Para mantener un control del entrenamiento, se confeccionó una lista de chequeo con todas las sesiones disponibles, donde cada persona marcó los días que entrenó $(9,10)$.

\section{Análisis estadístico}

Se aplicó la prueba de Shapiro Wilk para establecer la distribución de normalidad de las variables del estudio, lactato, Pimax y TM6M. Para los que poseen distribución no normal se utilizó la prueba no paramétrica de U de Mann-Whitney para determinar si la diferencia previa y posterior al EMI es significativa, estableciendo el nivel de significancia en un p valor $<0,05$. Se empleó el software GraphPad Prism versión 6.0. 


\section{Consideraciones éticas}

Todos los participantes firmaron el consentimiento informado, y los procedimientos fueron aprobados por el Comité de Ética de la Universidad Santo Tomás. El estudio consideró los aspectos éticos establecidos en la Declaración de Helsinki.
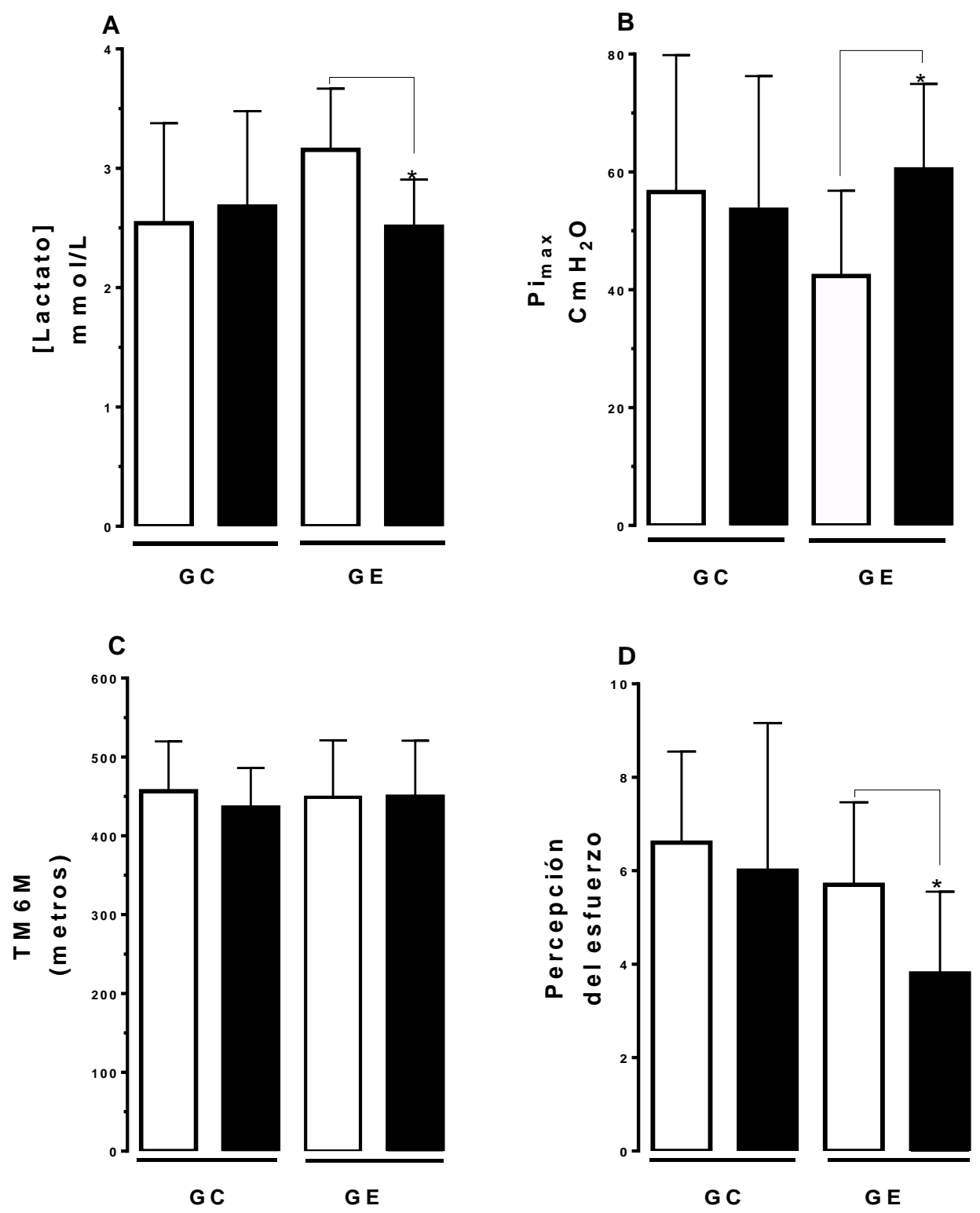

Figura 2. Efecto del entrenamiento con válvula umbral en el grupo entrenamiento vs. control

Los valores absolutos antes (barras blancas) y después (barras negras) de 4 semanas de entrenamiento. GC (grupo control), GE (grupo entrenado). A y B evidencian los cambios en la concentración de lactato y Pimax respectivamente para ambos grupos. C y D muestran los cambios en la distancia recorrida y percepción del esfuerzo, respectivamente. Promedios y desviación estándar; *p<0,05 según prueba de U de Mann-Whitney. 
Tabla 2. Efectos del programa de entrenamiento con válvula umbral para el grupo de entrenamiento vs. control

\begin{tabular}{|c|c|c|c|c|c|c|c|c|}
\hline \multirow{2}{*}{ Variable } & \multicolumn{4}{|c|}{ Grupo control } & \multicolumn{4}{|c|}{ Grupo entrenado } \\
\hline & $\begin{array}{c}\text { Pre- } \\
\text { entrenamiento }\end{array}$ & $\begin{array}{l}\text { Post- } \\
\text { entrenamiento }\end{array}$ & $\begin{array}{c}\% \text { de } \\
\text { cambio }\end{array}$ & valor $p$ & $\begin{array}{c}\text { Pre- } \\
\text { entrenamiento }\end{array}$ & $\begin{array}{l}\text { Post- } \\
\text { entrenamiento }\end{array}$ & $\begin{array}{c}\% \text { de } \\
\text { cambio }\end{array}$ & valor $p$ \\
\hline FC, lpm & $79,80 \pm 8,23$ & $78,60 \pm 7,30$ & $-1,2 \pm 1,37$ & 0,0058 & $67,67 \pm 8,03$ & $69,78 \pm 6,38$ & $-5,5 \pm 2,61$ & $<0,0001$ \\
\hline \multicolumn{9}{|c|}{ Presion arterial, $\mathrm{mmHg}$} \\
\hline Sistolica & $118,6 \pm 8,9$ & $111 \pm 9,61$ & $-2,1 \pm 2,66$ & 0,0400 & $119,33 \pm 8,32$ & $119,44 \pm 6,42$ & $-3,5 \pm 2,75$ & 0,0030 \\
\hline Diastolica & $63,8 \pm 4,14$ & $60,6 \pm 3,78$ & $-1,0 \pm 4,69$ & 0,6465 & $71,3 \pm 4,58$ & $66,11 \pm 7,81$ & $-3,6 \pm 6,55$ & 0,0739 \\
\hline PAM & $80,87 \pm 2,18$ & $78 \pm 2,74$ & $-1,5 \pm 3,17$ & 0,1535 & $87,23 \pm 4,23$ & $83,89 \pm 6,59$ & $-3,7 \pm 3,50$ & 0,0040 \\
\hline $\begin{array}{l}\text { Doble } \\
\text { producto, UA }\end{array}$ & $1 \pm 1$ & $1 \pm 1$ & $-4,4 \pm 5,07$ & 0,0320 & $1 \pm 1$ & $1 \pm 1$ & $-9,3 \pm 5,3$ & $<0,0001$ \\
\hline $\begin{array}{l}\text { Lactato, } \\
\mathrm{mmol} / \mathrm{L}\end{array}$ & $2,54 \pm 0,83$ & $2,88 \pm 1,13$ & $-2,1 \pm 2,66$ & 0,0400 & $3,16 \pm 0,51$ & $2,51 \pm 0,39$ & $-3,5 \pm 2,75$ & 0,0030 \\
\hline Pimax, $\mathrm{cmH}_{2} \mathrm{O}$ & $56,6 \pm 23,24$ & $53,80 \pm 22,48$ & $-1,0 \pm 4,69$ & 0,6465 & $42,11 \pm 14,5$ & $60,44 \pm 14,4$ & $-3,6 \pm 6,55$ & 0,0739 \\
\hline TM6M, metros & $458,4 \pm 66,63$ & $435,8 \pm 50,32$ & $-1,5 \pm 3,17$ & 0,1535 & $448,8 \pm 72,3$ & $449,6 \pm 70,9$ & $-3,7 \pm 3,50$ & 0,0040 \\
\hline $\begin{array}{l}\text { Percepción del } \\
\text { esfuerzo }\end{array}$ & $6,2 \pm 2,68$ & $5,8 \pm 3,56$ & $-4,4 \pm 5,07$ & 0,0320 & $5,56 \pm 1,81$ & $4 \pm 2$ & $-9,3 \pm 5,3$ & $<0,0001$ \\
\hline
\end{tabular}

Los datos se expresan por medio \pm SD. PAM (Presión arterial media); FC (Frecuencia cardiaca); TM6M (test marcha 6 minutos); UA = Unidades arbitrarias.

$*$ Diferencia significativa entre el entrenamiento previo y posterior (valor $\mathrm{p}<0,05$ )

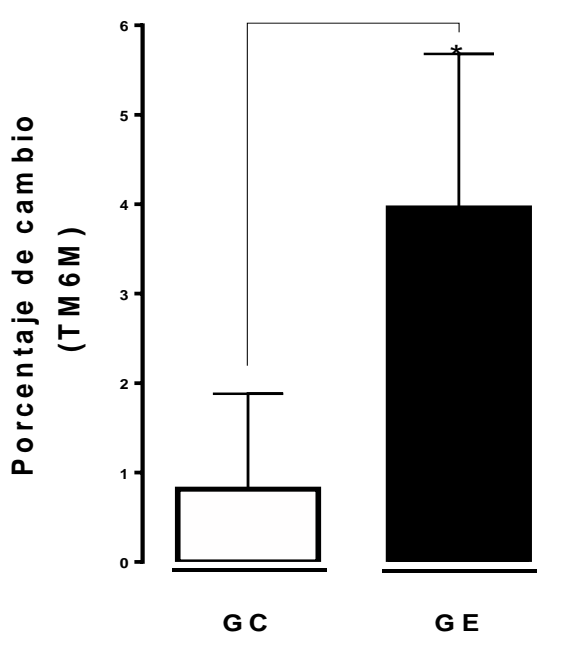

Figura 3. Porcentaje de cambio en los metros recorridos después del entrenamiento con válvula umbral

El test de marcha de 6 minutos (TM6M), muestra el porcentaje de cambio en metros recorridos, grupo control (barra blanca) y grupo entrenamiento (barra negra). Promedios y desviación estándar; ${ }^{*} \mathrm{p}<0,05$ según prueba de U de Mann-Whitney

\section{Entrenamiento de la musculatura inspiratoria}

Se evaluó el efecto del EMI sobre la concentración de lactato, Pimax, escala de percepción del esfuerzo y el TM6M. Como muestra la Figura 2 (A, B y D), los valores del primero evidenciaron una reducción en el GE, 3,16 $\pm 0,51$ a 2,5 $\pm 0,39$ con un valor de $\mathrm{p}<0,049$, el segundo presentó un incremento en el GE, $42,1 \pm 14,57$ a $60,44 \pm 14,47$ con un valor de $\mathrm{p}<0,017$. El tercero disminuyó en ambos grupos, sin embargo, se observan cambios significativos en el GE, $5,56 \pm 1,81$ a $4 \pm 2$ con un valor de $p<0,043$. Por último, el TM6M, no presentó cambios en sus valores absolutos en ningún grupo (Figura 2) (C). No obstante, se puede observar una diferencia de cambio porcentual entre el GE y GC, con un valor de $\mathrm{p}<0,031$, (Figura 3). El GC no mostró cambios en ninguna de sus variables, (Tabla 2).

\section{Discusión}

El entrenamiento de la musculatura inspiratoria es un método específico que contribuye al incremento de fuerza y resistencia de la musculatura respiratoria. En este sentido, esta musculatura posee características similares a la fibra muscular esquelética, por tanto, debería responder al entrenamiento de forma similar al músculo locomotor, aplicando una carga fisiológica adecuada. No obstante, existe cierta controversia en la dosificación del ejercicio (volumen e intensidad) necesario para generar adaptaciones musculares y funcionales en esta población(11). 
Sumado a lo anterior, un incremento en las demandas metabólicas de los músculos respiratorios no entrenados afectaría la capacidad funcional. En esta línea, Moreno(12), establece la relación entre la fuerza muscular inspiratoria con el $\mathrm{VO}_{2 \text { máx, }}$ señalando que un correcto EMI influye en el metabolismo energético de estos, generando menor demanda de oxígeno, asociado a una menor acumulación de lactato posterior a un esfuerzo físico; lo que se traduce en una mejor capacidad funcional. Esto concuerda con los resultados presentados en este estudio, que muestra una tendencia estadísticamente significativa sobre el lactato sanguíneo $(\mathrm{p}<0,049)$ entre el GE comparado con el GC. Los hallazgos descritos guardan relación con los reportados por Tong, et al.(13), quienes estudiaron en 30 deportistas universitarios la función de los músculos inspiratorios después de un EMI, argumentando una eficiencia en el barrido láctico asociado a las adaptaciones de fuerza $\mathrm{y}$ resistencia del diafragma. Dicho evento podría explicarse por la función del equilibrio ácido-base, optimizando el control de la acidosis metabólica mediante un eficiencia del funcionamiento de los quimiorreceptores que modulan los mecanismos de frecuencia y amplitud en el patrón respiratorio, estableciendo un control en el equilibrio ácido-base en condiciones de ejercicio(14).

Dos posibles mecanismos pueden explicar el menor aumento de la concentración de lactato desde un estado basal post ejercicio(15):

1) Después de entrenar la musculatura inspiratoria hay una reducción de la demanda energética debido al menor trabajo respiratorio.

2) Los músculos entrenados usan más lactato como combustible para su propia actividad.

El primer mecanismo ocurre por la vinculación del lactato con la fatiga, debido a que existen estudios como el de Archiza, et al.,(16), sus resultados muestran que con el EMI se mejora el suministro de oxígeno y sanguíneo en la musculatura locomotora, retrasando la aparición de la fatiga. Lo anterior es enfatizado por Rodríguez, et al., quien presenta resultados similares en 20 mujeres AM, evidenciando una mejor capacidad aeróbica y retrasando la aparición de la fatiga(17). La conclusión de estos autores es que al postergar la depleción de glucógeno, se pospone la fatiga, definiéndose ésta como "la incapacidad de seguir manteniendo una fuerza muscular apropiada al esfuerzo, llevando a disminuir la performance del sujeto"(18). Este concepto de fatiga en la musculatura respiratoria está ligado a procesos de activación de los mecanorreceptores y el metabolorreflejo. Lo anterior, se explica en la fuerza contráctil asociada con el ejercicio, se presume que aumenta la deformación mecánica del diafragma e incrementan los metabolitos asociados a la progresión del esfuerzo como: la adenosina, ácido láctico, fosfato y cationes, vinculados a fibras aferentes III y IV respectivamente(19), provocando una respuesta simpática asociada a una vasocontricción de la musculatura locomotora, intensificando la fatiga de la musculatura activa e incrementando la percepción del esfuerzo como lo señala $\operatorname{Romer}^{(20) .}$

El segundo mecanismo se explica mediante la adaptación de los músculos respiratorios, incrementando la capacidad de metabolizar lactato y al mismo tiempo incrementar la remoción de lactato circulante en la sangre. Esto concuerda con una investigación en animales donde se demostró que se puede consumir más lactato en ejercicio intenso, llevando así a una mayor metabolización de este sustrato, y por lo tanto disminuyendo su concentración en sangre. Resultados similares fueron obtenidos por Mehani(18), argumentando una disminución del metabolorreflejo, atenuando el fenómeno de vasoconstricción en los músculos de las extremidades inferiores.

La mitad de la energía requerida por el diafragma es derivada del metabolismo de los carbohidratos, donde principalmente es utilizado el lactato, por lo tanto al entrenar el diafragma, se estará utilizando más lactato como sustrato energético. Lo anterior se relaciona íntimamente con el Pimax, que se incrementó debido al entrenamiento, fortaleciendo la musculatura accesoria de la respiración y al mismo diafragma; ayudando al proceso de ventilación, que posterga la aparición de la fatiga, y dilata el proceso de redistribución de sangre al estar el sujeto bajo una alta intensidad de ejercicio(21). En este estudio los valores del Pimax, también presentan mejoras en comparación al GC ( $\mathrm{p}<0,017)$, dichos resultados se asemejan a los de Kilding y Karadalli(22). En cuanto a la rutina de entrenamiento, siguió lo propuesto por $\operatorname{Holm}^{(23)}$, entrenar cinco veces por semana, ya que esto se ajustaba al itinerario de los sujetos de prueba. Se decidió utilizar una rutina sencilla de 30 repeticiones, una vez por día, todo esto por un total de 4 semanas(10). 
Acerca del TM6M, se puede evidenciar que no hay significancia en los valores obtenidos antes $\mathrm{y}$ después de la intervención entre el GE y el GC. Lo anterior se puede ese explicar ya que el TM6M, es una prueba de campo que pasa a ser subjetiva, ya sea por la motivación y estado físico general de la persona estudiada, como también, el trato que el evaluador a cargo le dé al sujeto. Sin embargo, al normalizar y analizar las diferencias de variación entre los grupos, se puede observar los cambios esperados, asociado a la ganancia en los metros recorridos post intervención. Esto se puede responder al vincular el retraso en la aparición de la fatiga periférica, descrita en los párrafos anteriores.

Por último, la percepción del esfuerzo también presentó modificaciones en GE, disminuyendo sus valores $(p<0,043)$. Los cuales condicen con Figueiredo, et al.(5), debido a que el entrenamiento al que fue sometido el grupo experimental tiene como objetivo la postergación de la aparición de la fatiga y por ello se espera una disminución en la percepción del esfuerzo. No obstante, es importante que el sujeto al que se administra esta escala esté familiarizado con ella, ya que diversos factores como los sicológicos y/o emocionales pueden influir entre en la fatiga reportada por cada individuo(23).

\section{Conclusiones}

Los datos obtenidos en este estudio no evidencian cambios favorables en los metros recorridos después de la intervención, hecho que se traduce en la ausencia de modificaciones de la CF. Sin embargo, el EMI mostro una disminución sobre la concentración de lactato después del programa de ejercicio, lo que podría identificar un retraso en la aparición de la fatiga, evitando la competitividad energética entre la musculatura respiratoria y locomotora. De acuerdo con los resultados alcanzados en este estudio se sugiere incorporar un EMI en los programas de ejercicio con orientación en salud, con el fin de proporcionar estrategias simples y aplicables que permitan mejorar la CF de las personas AM.

A futuro se recomienda agregar periodos más largos de entrenamiento para evaluar si la respuesta observada se modifica en el tiempo, del mismo modo es esperable incorporar una mayor cantidad de participantes con distintas condiciones.
Conflicto de intereses: Los autores declaramos no tener conflictos de intereses.

\section{Referencias}

1. SENAMA Ministerio de Desarrollo Social. Política Integral de Envejecimiento Positivo para Chile 2012-2025. Santiago, Chile: Ministerio de Desarrollo Social; 2016.

2. Palma-Candia O, Hueso-Montoro C, Ortega-Valdivieso A, Montoya-Juárez R, Cruz-Quintana F. Factores sociodemográficos y de participación social relacionados con el bienestar psicológico en adultos mayores en la región de Magallanes, Chile. Revista médica de Chile. 2016;144(10):1287-1296.

3. Tapia CP, Valdivia-Rojas Y, Varela HV, Carmona AG, Iturra VM, Jorquera MC.Rates of frailty among older people ascribed to Chilean primary care clinics. Rev Med Chil. 2015;143(4):459-66.

4. Mafi F, Biglari S, Afousi AG, Gaeini AA. Epicatechin Supplementation and Resistance Training-Induced Improvement of Muscle Strengthand Circulatory Levels of Plasma Follistatin and Myostatin in Sarcopenic Older Adults. J Aging Phys Act. 2018;9:1-27.

5. Figueiredo PHS, Lima MMO, Costa HS, Martins JB, Flecha OD, Gonçalves PF, et al. Effects of the inspiratory muscle training and aerobic training on respiratory and functional parameters, inflammatory biomarkers, redox status and quality of life in hemodialysis patients: A randomized clinical trial. PLoS One. 2018;13(7):e0200727. doi: 10.1371/journal.pone.0200727

6. Romer LM, Miller JD, Haverkamp HC, Pegelow DF, Dempsey JA. Inspiratory muscles do not limit maximal incremental exercise performance in healthy subjects. Respir Physiol Neurobiol. 2007;156(3):353-61.

7. Raissuni Z, Roul G. Comparison of the long-term reproducibility of the walk test and of exercise peak oxygen consumption in patients with preserved exercise capacity. Acta Cardiol. 2018;73(2):155-162.

8. Szeinberg A, Marcotte JE, Roizin H, Mindorff C, England S, Tabachnik E, Levison $H$. Normal values of maximal inspiratory and expiratory pressures with a portable apparatus in children, adolescents, and young. adults Pediatr Pulmonol. 1987;3:255-8.

9. Alvarenga G, Charkovski S, Santos L, Silva M, Tomaz G, Gamba H. The influence of inspiratory muscle training combined with the Pilates method on lung function in elderly women: A randomized controlled trial. Clinics (São Paulo). 2018;73:e356.

10. González M. Efectos del entrenamiento de la musculatura respiratoria sobre el rendimiento. Revisión bibliográfica. Rev Andaluza Med del Deport. 2012;5(4):163-70.

11. Kulnik ST, Rafferty GF, Birring SS, Moxham J, Kalra L. A pilot study of respiratory muscle training to improve cough effectiveness and reduce the incidence of pneumonia in acute stroke: study protocol for a randomized controlled trial. Trials. 2014;15:123.

12. Moreno AM, Toledo-Arruda AC, Lima JS, Duarte CS, Villacorta H, Nóbrega ACL. Inspiratory Muscle Training Improves Intercostaland Forearm Muscle Oxygenation in Patients With Chronic Heart Failure: Evidence of the Origin of the Respiratory Metaboreflex. J Card Fail. 2017;23(9):672-679. 
13. Tong TK, Fu FH, Chung PK, Eston R, Lu K, Quach B, et al. The effect of inspiratory muscle training on high- intensity, intermittent running performance to exhaustion. Appl Physiol Nutr Metab. 2008;681:671-81.

14. Peters CM, Welch JF, Dominelli PB, Molgat-Seon Y, Romer LM, McKenzie DC, Sheel AW. Influence of inspiratory resistive loading on expiratory muscle fatigue in healthy humans. Exp Physiol. 2017;102(9):1221-1233.

15. Spengler CM, Roos M, Laube SM, Boutellier U. Decreased exercise blood lactate concentrations after respiratory endurance training in humans. Eur J Appl Physiol Occup Physiol. 1999;79(4):299-305.29.

16. Archiza B, et al. Effects Of Inspiratory Muscle Training In Professional Women Football Players: A Randomized ShamControlled Trial. J Sports Sci. 2018;36(7):771-780.

17. Rodrigues GD, Gurgel JL, Gonçalves TR, da Silva-Soares PP. Inspiratory muscle training improves physical performance and cardiac autonomic modulation in older women. Eur J Appl Physiol. 2018;118(6):1143-1152.
18. Mehani SH. Comparative study of two different respiratory training protocols in elderly patients with chronic obstructive pulmonary disease. Clin Interv Aging. 2017;12:1705-1715.

19. Allen DG, Lamb D, Westerblad H. Skeletal muscle fatigue: cellular mechanisms. Physiological reviews, 2008, vol. 88, no 1, p. 287-332.

20. Romer LM, Lovering AT, Haverkamp HC, Pegelow DF, Dempsey JA. Effect of inspiratory muscle work on peripheral fatigue of locomotor muscles in healthy humans. J Physiol. 2006;571(Pt 2):425-39.

21. Griffiths LA, McConnell AK. The influence of inspiratory and expiratory muscle training upon rowing performance.Send to Eur J Appl Physiol. 2007;99(5):457-66.

22. Kilding AE1, Brown S, McConnell AK.Inspiratory muscle training improves 100 and $200 \mathrm{~m}$ swimming performance. Eur J Appl Physiol. 2010;108(3):505-11.

23. Holm P, Sattler A, Fregosi RF. Endurance training of respiratory muscles improves cycling performance in fit young cyclists. BMC Physiol. 2004;4:9. 